\title{
Algebraicity of Hodge Loci for Variations of Hodge Structure
}

\author{
Eduardo Cattani and Aroldo Kaplan
}

\begin{abstract}
These notes should be seen as a companion to $[\mathbf{8}]$, where the algebraicity of the loci of Hodge classes is proven without appealing to the Hodge conjecture. We give explicit detailed proofs in the case of variations of Hodge structures over curves and surfaces which, we hope, help clarify the arguments in [8], as well as some generalizations, consequences and conjectures based on those results.
\end{abstract}

\section{Introduction}

The properties of Hodge classes, i.e., rational or integral cohomology classes of pure Hodge type (p,p), in families of varieties have been one of Griffith's concerns since his early papers on Periods of Integrals. His ideas led in particular to the notion of abstract Hodge structure and of variations thereof, which became essential for the subject. For even in the case of geometric families some arguments require intermediate variations that are not necessarily geometric. Indeed, the results discussed below provide a good example of this phenomenon.

In [39], A. Weil comments: "It seems to be a known fact (cf. e.g. Griffiths, passim) that to impose a Hodge class upon a manifold with complex structure imposes upon its local moduli a holomorphic condition. In other words, if we have a family $\left\{M_{t}\right\}$ of such manifolds depending holomorphically upon local complex parameters $t$, and if one of them, say $M_{0}$, carries a Hodge class $\omega$, those $M_{t}$ near $M_{0}$ for which $\omega$ is still a Hodge class make up a complex-analytic subfamily of $\left\{M_{t}\right\}$." And later: "From the point of view of algebraic geometry [...] One may now ask whether imposing a certain Hodge class upon a generic member of an algebraic family of polarized algebraic varieties amounts to an algebraic condition upon the parameters".

A positive answer to Weil's question would follow from the rational Hodge conjecture and thus the question arose whether the statement could be proved or disproved independently of the Hodge conjecture. Griffiths expected that variations of Hodge structures would be the appropriate setting to study the problem, and Schmid's Orbit Theorems the appropriate tool. His letters on the subject led Deligne to prove the corresponding statement for variations over curves [15]. As

2000 Mathematics Subject Classification. Primary 14C30, 14G07, 32G20 .

Partially supported by CONICET (Argentina). 
expected, his argument made essential use of the $S L_{2}$-orbit theorem which, at the time, was only known for variations over curves.

Following the development of the multivariable $S L_{2}$ theory, it became possible to attack Weil's question in full generality. This was done in [8]. In this article we review the results of that paper, discuss some generalizations, consequences and conjectures, and present simplified proofs of the crucial local statement for variations in one and two parameters. This has several advantages which we hope readers will find useful: the proof in the curve case follows roughly along the lines of Deligne's argument in [15] and serves not only as an inductive step but also as an excellent introduction to the ideas used in the general case; it clarifies the role played by the different real splittings of the limiting mixed Hodge structure; the inductive step becomes much clearer, free of the substantial notational burden of the general case; the bivariate case already provides an example where full monodromy invariance fails, a phenomenon somewhat unexpected given the curve case.

The result that answers Weil's question in the affirmative, is

THEOREM 1.1. In a smooth algebraic variety parametrizing a polarizable variation of Hodge structure, the locus where a locally flat integral section has a determination of pure type $(p, p)$, is algebraic.

The variety in question is not required to be complete nor the section to be single-valued. In these cases, the conclusion follows easily from Griffiths's basic theorems on variations. It is at infinity, where the variation degenerates and infinitely many determinations may appear, where most of the problems occur. In turn, these lead to local properties of the locus at normal crossings which are of independent interest.

\section{Results, consequences and generalizations}

In what follows we assume that the reader is familiar with the basic notions of Hodge structures, variations of Hodge structure, classifying spaces and period maps. We refer to $[\mathbf{2 2}, \mathbf{2 3}, \mathbf{2 5}]$ for further details. To set the notation, let $\left(\mathcal{V}_{\mathbb{Z}}, \mathcal{F}\right)$ be an (integral) variation of Hodge structure of weight $2 p$ over a complex manifold $S$. Here $p$ is a non-negative integer, $\mathcal{V}_{\mathbb{Z}}$ is a local system of free $\mathbb{Z}$-modules over $S$ and $\mathcal{F}$ a decreasing filtration of the holomorphic vector bundle $\mathcal{V}=\mathbb{C} \otimes \mathcal{V}_{\mathbb{Z}}$ by holomorphic subbundles

$$
\mathcal{V}=\mathcal{F}^{0} \supseteq \mathcal{F}^{1} \supseteq \cdots \supseteq \mathcal{F}^{2 p} \supseteq 0
$$

such that for all $q$

$$
\nabla \mathcal{F}^{q} \subset \Omega_{S}^{1} \otimes \mathcal{F}^{q-1},
$$

and

$$
\mathcal{V}=\mathcal{F}^{q} \oplus \overline{\mathcal{F}}^{2 p-q+1}
$$

where $\nabla$ is the flat Gauss-Mannin connection and conjugation is relative to $\mathcal{V}_{\mathbb{R}}=$ $\mathcal{V}_{\mathbb{Z}} \otimes \mathbb{R}$. We will identify throughout the vector bundle $\mathcal{V}$ with its associated sheaf of sections.

Setting $\mathcal{V}^{a, b}=\mathcal{F}^{a} \cap \overline{\mathcal{F}}^{b}$, the Hodge decomposition

$$
\mathcal{V}=\bigoplus_{a+b=2 p} \mathcal{V}^{a, b}
$$


is a smooth grading of $\mathcal{F}$. Let $\mathcal{C}$ be the smooth section of $\operatorname{End}(\mathcal{V})$ acting on $\mathcal{V}^{a, b}$ as multiplication by $i^{a-b}$.

We assume that the variation is polarizable, meaning that for some fixed locally constant symmetric bilinear form $\mathcal{Q}$ on $\mathcal{V}_{\mathbb{Z}}$, the induced Hermitian form on $\mathcal{V}$

$$
h(u, v)=\mathcal{Q}(\mathcal{C} u, \bar{v})
$$

is positive definite and makes the Hodge decomposition orthogonal. This metric is generally not flat.

The geometric case is that of an algebraic family $\mathcal{X} \rightarrow S$ of smooth, polarized projective varieties. It gives rise to a polarizable variation of Hodge structure, of weight $k$, on the local systems of primitive cohomology of degree $k$. The flat bundle of lattices corresponding to the sheaf $\mathcal{V}_{\mathbb{Z}}$ has fibers $\left(\mathcal{V}_{\mathbb{Z}}\right)_{s} \cong H_{p r}^{k}\left(X_{s}, \mathbb{Z}\right)$, and $\mathcal{F}_{s}$ is the usual Hodge filtration. For $k=2 p$, the elements of

$$
H^{2 p}\left(X_{s}, \mathbb{Z}\right) \cap H_{p r}^{p, p}\left(X_{s}\right)
$$

are the Hodge classes of codimension $p$ of $X_{s}$.

Recall that the flat sections of $\mathcal{V}_{\mathbb{Z}}$, or of $\mathcal{V}$, on $S$ will generally be multivalued: the corresponding monodromy representation of the fundamental group of $S$ is naturally associated to the local system $\mathcal{V}_{\mathbb{Z}}$. Since the Hodge filtration is not flat, whether a locally flat integral section $u$ is of pure type $(p, p)$ at some point $s \in S$ will depend on the point, as well as on the particular determination of $u$ at that point.

THEOREM 2.1. Let $\left(\mathcal{V}_{\mathbb{Z}}, \mathcal{F}\right)$ be a polarizable variation of Hodge structure of weight $2 p$ over an algebraic variety $S$ and let $u$ be a locally constant section of $\mathcal{V}_{\mathbb{Z}}$. Then, the set $S(u)$ of points in $S$ where some determination of $u$ is of pure type $(p, p)$, is an algebraic subvariety of $S$.

The rational span of a Hodge class is a special case of Hodge substructure of a Hodge structure. This is a rational suspace $U_{\mathbb{Q}}$ such that

$$
U=U_{\mathbb{Q}} \otimes \mathbb{C}=\bigoplus_{a+b=k} U \cap H^{a, b} .
$$

For example, in the geometric case, if $Z \subset X_{s}$ is an algebraic subvariety, the subspaces

$$
\operatorname{ker}\left\{i^{*}: H^{k}\left(X_{s}, \mathbb{Q}\right) \longrightarrow H^{k}\left(X_{s} \backslash Z, \mathbb{Q}\right)\right\}
$$

where $i: X_{s} \backslash Z \hookrightarrow X_{s}$ is the inclusion map, are Hodge substructures of $H^{k}\left(X_{s}, \mathbb{C}\right)$. Theorem 2.1 may be generalized as follows.

THEOREM 2.2. Let $\left(\mathcal{V}_{\mathbb{Z}}, \mathcal{F}\right)$ be a polarizable variation of Hodge structure over an algebraic variety $S$ and let $U_{\mathbb{Q}} \subset\left(\mathcal{V}_{\mathbb{Q}}\right)_{s_{0}}, s_{0} \in S$, be a rational subspace. Then, the locus where some flat translate of $U_{\mathbb{Q}}$ is a Hodge substructure, is an algebraic subvariety of $S$.

In the proof of Theorem 2.1, one considers the whole Hodge locus $\mathcal{L}$ of a variation, instead of that defined by just one section; this is often called the (integral) Noether-Lefschetz locus. If $\left(\mathcal{V}_{\mathbb{Z}}, \mathcal{F}\right)$ is a variation of polarized Hodge structure of weight $2 p$ on a complex manifold $S$, its integral Hodge locus is the subset of the bundle $\mathcal{F}^{p}$ defined by

$$
\mathcal{L}=\left\{(s, u): s \in S, u \in \mathcal{F}_{s}^{p} \cap\left(\mathcal{V}_{\mathbb{Z}}\right)_{s}\right\} .
$$


If $\mathcal{Q}$ is a fixed polarizing form of $\left(\mathcal{V}_{\mathbb{Z}}, \mathcal{F}\right)$ and $K$ any positive number, define

$$
\mathcal{L}_{K}=\{(s, u) \in \mathcal{L}, \mathcal{Q}(u, u) \leq K\}
$$

Theorem 2.1 then follows from

THEOREM 2.3. If $S$ is algebraic, then $\mathcal{L}_{K}$ is an algebraic subvariety of the bundle $\mathcal{F}^{p}$, finite over $S$.

In her beautiful exposition on Hodge loci [37], C. Voisin calls this finiteness "intriguing" because it is stronger than what is implied by the Hodge conjecture and against what it is predicted by geometry. For in the geometric case, it implies that there are only finitely many components of the locus of primitive Hodge classes with of Hodge lenght $\leq K$, something expected for rational classes, but not for the integral ones, as explained by the Soulé-Voisin examples [36] derived from Kollar's counterexamples to the integral Hodge conjecture.

We leave the reduction to a local statement and the proof for variations in one or two parameters to the following sections, and now mention some further implications, generalizations and refinements.

In the geometric case, if the family is defined over a number field, the Hodge Conjecture would imply that the Hodge loci would be defined over the same field, an assertion which is not accessible to the methods of [8]. The following partial result is due to $\mathrm{C}$. Voisin $[\mathbf{3 7}, \mathbf{3 8}]$.

THEOREM 2.4. Let $X_{t}$ be a family of algebraic varieties parametrized by a quasi-projective base $B, \alpha$ a Hodge class and $B_{\alpha} \subset B$ be a component of the Hodge locus of $\alpha$. Suppose that any locally constant Hodge substructure $L \subset H^{2 k}\left(X_{t}, \mathbb{Q}\right)$, $t \in B_{\alpha}$, is of pure type $(k, k)$. Then $B_{\alpha}$ is defined over $\mathbb{Q}$ and its translates under $\operatorname{Gal}(\overline{\mathbb{Q}} / \mathbb{Q})$ are again components of the Hodge locus.

REMARK 2.5. An obvious question in the context of the subject of these proceedings concerns the special properties of Hodge loci for variations associated with non-classical Mumford-Tate domains $D$, i.e., those whose period map takes values in such $D$. In the classical case the asymptotic behavior of the period map is described by the Nilpotent and $S L_{2}$ Orbit Theorems. Although the approximating nilpotent orbit in the ambient classifying space can be chosen to lie in $D$, for $S L_{2}$ orbits this seems to hold only in special cases, as Griffiths points out in the last of his CBMS lectures. This is because in the $S L_{2}$ representation associated to the nilpotent orbit by Schmid's Theorem the semisimple element is only defined over $\mathbb{R}$. Although the splitting of the limiting mixed Hodge structure defined by the $S L_{2}$-orbit looks transcendental, it actually depends algebraically on the nilpotent orbit data (see $[\mathbf{1 1}, \mathbf{1 7}])$.

Analogous problems arise when one tries to extend the results of [8] to NoetherLefschetz loci in non-abelian Hodge Theory [35] and in D-branes of Calabi-Yau [19].

For partial results about the asympotics of period maps on Mumford-Tate domains, see the talks of Griffiths and Pearlstein and also [27].

In other directions, Brosnan, Pearlstein and Schnell generalized Theorem 2.1 to variations of Mixed Hodge structure. In [7] they prove

THEOREM 2.6. Let $\mathcal{V}$ be an integral variation of graded polarized mixed Hodge structure on a Zariski-open subset $S$ of a complex manifold $\bar{S}$. Assume that $\mathcal{V}$ is admissible with respect to $\bar{S}$. Then each component of the locus of Hodge classes extends to an analytic space, finite and proper over $S$. 
This result uses the results of the first two authors of [7] on the locus of zeroes of normal functions, themselves based on Theorem 2.1. With $S$ and $\bar{S}$ as above, Brosnan and Pearlstein prove:

THEOREM 2.7. Let $\nu$ be an admissible higher normal function on $S$, that is, an admissible extension of $\mathbb{Z}(0)$ by a variation of polarized Hodge structure of negative weight, and let $Z$ be its zero-locus. Then $\bar{Z} \subset \bar{S}$ is an analytic subset. Moreover, if $S$ is quasiprojective, then $Z$ is algebraic.

Theorem 2.1 implies that other special varieties defined by Hodge loci are $a$ priori algebraic. For specific cases and applications to normal functions we refer to $[4,20,21,28,31]$. For applications to the classical Lefschetz-Noether locus of families, see $[\mathbf{3}, \mathbf{2 1}, \mathbf{2 9}, \mathbf{3 2}, \mathbf{3 3}]$.

\section{Reduction to a Local Statement}

In this section we will reduce the proof of Theorem 2.1 to a local statement, namely Theorem 3.3, and then deduce Theorem 2.2 from it. We start with the following

LEMma 3.1. $S(u)$ is an analytic subvariety of $S$.

Proof. Let $s_{0} \in S(u)$ and suppose $v$ is a determination of $u\left(s_{0}\right)$ such that

$$
v \in\left(\mathcal{V}_{\mathbb{Z}}\right)_{s_{0}} \cap \mathcal{V}_{s_{0}}^{p, p}
$$

For a simply connected neighborhood $\mathcal{N}$ of $s_{0}$ in $S$,

$$
S(u) \cap \mathcal{N}=\bigcup_{\gamma \in \Gamma} \Sigma(\gamma v)
$$

where $\Gamma$ denotes the monodromy group of the variation acting on the fibre over $s_{0}$ and $\Sigma(\gamma v)$ the locus of points in $\mathcal{N}$ for which the parallel translate of $\gamma v$, along a path contained in $\mathcal{N}$, remains of type $(p, p)$.

Since

$$
\mathcal{V}_{\mathbb{Z}} \cap \mathcal{V}^{p, p}=\mathcal{V}_{\mathbb{Z}} \cap \mathcal{F}^{p} \cap \overline{\mathcal{F}}^{p}=\mathcal{V}_{\mathbb{Z}} \cap \mathcal{F}^{p}
$$

the condition for the flat translate of a fixed $\gamma v$ to be of type $(p, p)$ at some point $s \in \mathcal{N}$ is holomorphic. Consequently, each $\Sigma(\gamma v)$ is analytic.

On the other hand, any element of type $(p, p)$ is fixed by the Weil operator $\mathcal{C}$, so that if $s \in \Sigma(\gamma v)$

$$
h_{s}(\gamma v, \gamma v)=\mathcal{Q}_{s}(\gamma v, \gamma v)=\mathcal{Q}_{s_{0}}(v, v)
$$

But, for $\mathcal{N}$ relatively compact, the Hodge norms $h_{s}, s \in \mathcal{N}$, are uniformly equivalent to the Hodge norm at $s_{0}$. Since the elements $\gamma v$ lie in a lattice this implies that $\Sigma(\gamma v)=\emptyset$ for all but finitely many of them and, consequently, $S(u)$ is an analytic subvariety of $S$.

To prove the algebraicity of $S(u)$, it is sufficient to consider the case when $S$ is quasi-projective; indeed, the complement of a divisor with normal crossings in a smooth projective variety $\bar{S}$. By Chow's theorem it will then be enough to show that the closure of $S(u)$ in $\bar{S}$ is analytic as well. In order to do this it is necessary to study $S(u)$ locally at the divisor $\bar{S} \backslash S$. To this effect, consider a polycylindrical neighborhood $\Delta^{r+l} \subset \bar{S}$ of a point $0 \in \bar{S} \backslash S$, such that $S \cap \Delta^{r+l}=\left(\Delta^{*}\right)^{r} \times \Delta^{l}$.

Then $\mathcal{V}_{\mathbb{Z}}, \mathcal{F}, \mathcal{Q}$ determines a variation on $\left(\Delta^{*}\right)^{r} \times \Delta^{l}$. While it is not possible to locally detect the determinations of the same multivalued flat section, according 
to the previous argument they will all have the same $\mathcal{Q}$-norm. Theorem 2.1 will therefore follow from the following statement.

THEOREM 3.2. Let $\mathcal{V}_{\mathbb{Z}}, \mathcal{F}, \mathcal{Q}$, define a polarized, integral variation of Hodge structure of weight $2 p$ over a product of punctured disks and disks $\left(\Delta^{*}\right)^{r} \times \Delta^{l}$. Let $\alpha$ be an integer and $K \subset \Delta^{l}$ a compact set. Then, there exist finitely many elements in the typical fiber

$$
v_{1}, \ldots, v_{N} \in V=\mathcal{V}_{s_{0}}
$$

and a neighborhood $\mathcal{U}$ of $0 \times K$ in $\Delta^{r} \times \Delta^{l}$ such that

(i) If $s \in \mathcal{U}, u \in\left(\mathcal{V}_{\mathbb{Z}}\right)_{s} \cap \mathcal{V}_{s}^{p, p}$, and $\mathcal{Q}_{s}(u, u)=\alpha$, then $u$ is the parallel translate of some $v_{j}$ along a path from $s_{0}$ to $s$;

(ii) Let $v_{1}, \ldots, v_{N}$ be the corresponding multivalued flat sections of $\mathcal{V}$ over $\left(\Delta^{*}\right)^{r} \times \Delta^{l}, S\left(v_{j}\right)$ the locus where some determination of that section is of type $(p, p)$. Then, the closures $\overline{S\left(v_{j}\right)} \subset \Delta^{r} \times \Delta^{l}$ are analytic subvarieties of this polydisk. $\pi_{1}\left(S\left(v_{j}\right)\right)$.

(iii) $v_{j}$ is invariant under the natural monodromy representation of

When the base is one-dimensional, either there are no integral $(p, p)$ elements of $\mathcal{Q}$-norm $K$ sufficiently near the puncture, or the locus of such elements will contain a full punctured disk, in which case the corresponding elements $v_{j}$ 's will be invariant by the full local monodromy. In the higher-dimensional case, however, (3.2)(iii) is all that one can expect.

We can make two simplifying assumptions entailing no loss of generality. First, we may assume that $l=0$; this amounts to considering a variation over a product of punctured disks extending holomorphically accross some of the punctures. We also suppose that the local monodromy, i.e. the action of $\pi_{1}\left(\left(\Delta^{*}\right)^{r}\right) \cong \mathbb{Z}^{r}$ on the typical fiber $V$, is unipotent. Indeed, by Borel's Monodromy Theorem (cf. [34]) it is necessarily quasi-unipotent and 3.2 will hold for a variation if it holds for its pull-back to any finite cover $\left(\Delta^{*}\right)^{r} \rightarrow\left(\Delta^{*}\right)^{r}$.

We now recall some results on the asymptotic behavior of a variation of Hodge structure, referring to $[\mathbf{1 0}, \mathbf{1 1}, \mathbf{1 2}, \mathbf{3 4}]$ for details.

Choosing coordinates $\mathbf{s}=\left(s_{1}, \ldots, s_{r}\right) \in\left(\Delta^{*}\right)^{r}$ we may, via parallel translation, view the filtration $\mathcal{F}_{\mathbf{s}}$ as a variable filtration in the typical fiber $V=\mathcal{V}_{\mathbf{s}_{0}}$, defined up to the action of the monodromy. Let $\mathcal{H}$ denote the upper half-plane; it covers the punctured disk $\Delta^{*}$ via $z \mapsto s=e^{2 \pi i z}$. Lifting the variation to the product $\mathcal{H}^{r}$ one obtains the period map:

$$
\Phi: \mathcal{H}^{r} \rightarrow \mathcal{D}
$$

with values on the appropriate classifying space of Hodge structures (on $V$, of weight $2 p$, appropriate Hodge numbers and polarized by $\left.Q=\mathcal{Q}_{\mathbf{s}_{0}}\right)$.

Explicitely, for $\mathbf{z}=\left(z_{1}, \ldots, z_{r}\right) \in \mathcal{H}^{r}, \Phi(\mathbf{z})$ is the parallel translate of the filtration $\mathcal{F}_{\mathbf{s}}$ to $V=\mathcal{V}_{\mathbf{s}_{0}}$, along the projection of any path in $\mathcal{H}^{r}$ joining $\mathbf{z}$ with $\mathbf{z}_{0}$; here

$$
\mathbf{s}=e^{2 \pi i \mathbf{z}},
$$

that is, $s_{j}=e^{2 \pi i z_{j}}$, for $j=1, \ldots, r$ and $\mathbf{z}_{0} \in \mathcal{H}^{r}$ is fixed so that $\mathbf{s}_{0}=e^{2 \pi i \mathbf{z}_{0}}$.

The map $\Phi$ is holomorphic, satisfies the horizontality condition

$$
\partial \Phi^{q} \subset \Omega^{1} \otimes \Phi^{q-1}
$$

as well as the period relation

$$
\Phi\left(\mathbf{z}+e_{j}\right)=\left(\exp N_{j}\right) \Phi(\mathbf{z})
$$


where $e_{j_{i}}=\delta_{j, i}$ and the $N_{j}^{\prime} s$ are the monodromy logarithms. The latter are mutually commuting infinitesimal isometries of $Q$ which preserve the rational structure on $V$.

According to Schmid's Nilpotent Orbit Theorem [34], one can write

$$
\Phi(\mathbf{z})=\exp \left(\sum_{j=1}^{r} z_{j} N_{j}\right) \cdot \Psi(\mathbf{s})
$$

for some holomorphic map

$$
\Psi: \Delta^{r} \rightarrow \check{\mathcal{D}}
$$

into the Zariski closure of $\mathcal{D}$ in the corresponding flag variety. Moreover, letting

$$
F:=\Psi(0)
$$

the nilpotent orbit

$$
\theta\left(z_{1}, \ldots, z_{r}\right)=\exp \left(\sum_{j=1}^{r} z_{j} N_{j}\right) \cdot F
$$

is again holomorphic and satisfies (1.7-8) -hence it is also the lifted period map of a variation over $\left(\Delta^{*}\right)^{r}$.

We must point out that the filtration $F \in \check{\mathcal{D}}$ depends on the choice of coordinates and only its orbit $\mathcal{F}_{\Phi}$ under the action of the abelian group

$$
\left\{\exp \left(\sum_{j=1}^{r} \mu_{j} N_{j}\right) ; \mu_{j} \in \mathbb{C}\right\} .
$$

is intrinsically associated to $\Phi$. We will refer to any filtration $F \in \mathcal{F}_{\Phi}$ as a limiting Hodge filtration.

Any nilpotent transformation $T \in \operatorname{End}(V), T^{k+1}=0$, has an associated weight filtration, $W(T)$ (see $[\mathbf{2 3}, \mathbf{3 4}]$ ). This is an increasing filtration,

$$
\{0\}=W_{-k-1}(T) \subset W_{-k}(T) \subset \cdots \subset W_{k}(T)=V
$$

characterized by the properties:

$$
T W_{\ell}(T) \subset W_{\ell-2}(T), \quad T^{\ell}: G r_{\ell}^{W(T)} \stackrel{\sim}{\rightarrow} G r_{-\ell}^{W(T)}
$$

The filtration $W(T)$ is defined over $\mathbb{Q}$ if $T \in \operatorname{End}\left(V_{\mathbb{Q}}\right)$. As shown in $[\mathbf{9}]$, if $\theta(\mathbf{z})$ is a nilpotent orbit and $\lambda_{1}, \ldots, \lambda_{r}$ are real and positive:

$$
W\left(\lambda_{1} N_{1}+\cdot+\lambda_{r} N_{r}\right)=W\left(N_{1}+\cdot+N_{r}\right) .
$$

We set

$$
W:=W\left(N_{1}+\cdots+N_{r}\right)[-2 p],
$$

where, as usual, $W[a]$ denotes the shifted filtration: $(W[a])_{\ell}=W_{\ell+a}$.

It is a consequence of the $S L_{2}$-Orbit Theorem that for any limiting Hodge filtration, the pair

$$
(W, F)
$$

is a mixed Hodge structure, polarized -in the sense of $[\mathbf{9}]$ and relative to the form $Q$ - by every $N=\lambda_{1} N_{1}+\cdot+\lambda_{r} N_{r}, \lambda_{j} \in \mathbb{R}_{>0}$.

In terms of the (lifted) period mapping, the finiteness statement (i) in Theorem 3.2 may now be restated as: 
THEOREM 3.3. Let $K>0$ be given, then for some $\beta>0$, the set $V_{\beta}$ consisting of all elements $v \in V_{\mathbb{Z}}$ with $Q(v, v) \leq K$ and such that $v \in \Phi^{p}(\mathbf{z})$ for some $\mathbf{z}$ with $\operatorname{Im}\left(z_{j}\right)>\beta,\left|\operatorname{Re}\left(z_{j}\right)\right| \leq 1$, is finite.

Moreover, suppose that the $(p, p)$-locus $S(v)$ of a flat integral section contains the origin $0 \in \Delta^{r}$ in its closure. Then

$$
v \in F^{p} \cap \bar{F}^{p} \cap W_{0}\left(N_{1}+\cdots+N_{r}\right)
$$

for some limiting Hodge filtration $F$.

Assuming Theorem 3.3, we will now proceed to prove (ii) and (iii) of Theorem 3.2. We fix an element $v \in V_{\mathbb{Z}}$ satisfying $Q(v, v) \leq K$, and such that the $(p, p)$-locus of the corresponding flat section,

$$
\Sigma=S(v)
$$

contains the origin $0 \in \Delta^{r}$ in its closure. $\Sigma$ is the projection onto $\left(\Delta^{*}\right)^{r}$ of the set

$$
\widetilde{\Sigma}=\left\{\mathbf{z} \in \mathcal{H}^{r}: v \in \Phi^{p}(\mathbf{z})\right\} .
$$

The group $G=O(Q, \mathbb{C})$ acts transitively and holomorphically on $\check{D}$, so we may write

$$
\Psi(\mathbf{s})=\exp \Gamma(\mathbf{s}) \cdot F
$$

where $F$ is as in (1.10) and $\Gamma:\left(\Delta^{*}\right)^{r} \longrightarrow \mathfrak{g}$ is a holomorphic map with values in the Lie algebra of $G$. We choose a specific lifting as follows. Let $\left\{I^{a, b}\right\}$ be the canonical bigrading of $V$ (cf. (4.1) below) associated to the mixed Hodge structure $(W, F)$. Then

$$
W_{\ell}=\bigoplus_{a+b \leq \ell} I^{a, b} \quad ; \quad F^{q}=\bigoplus_{a \geq q} I^{a, b} .
$$

Being a Lie algebra of endomorphisms of $V, \mathfrak{g}$ inherits a bigrading $\left\{I^{a, b} \mathfrak{g}\right\}$ in terms of which the isotropy subalgebra of $\mathfrak{g}$ at $F$ is $\mathfrak{h}=\bigoplus_{a \geq 0} I^{a, b} \mathfrak{g}$ and

$$
\mathfrak{g}=\mathfrak{h}+\mathfrak{b} \quad ; \quad \mathfrak{b}=\bigoplus_{a<0} I^{a, b} \mathfrak{g}
$$

Since $\Psi(0)=F$, we can choose the function $\Gamma(\mathbf{s})$ to be holomorphic, $\mathfrak{b}$-valued and such that $\Gamma(0)=0$. Note that $\mathfrak{b}$ is a graded nilpotent Lie algebra:

$$
\mathfrak{b}=\mathfrak{b}^{-1} \oplus \mathfrak{b}^{-2} \oplus \cdots \quad ; \quad \mathfrak{b}^{r}=\bigoplus_{a=r} I^{a, b} \mathfrak{g}
$$

The monodromy logarithms are $(-1,-1)$-morphisms of the mixed Hodge structure $(W, F)$. Hence, they satisfy $N_{j}\left(I^{a, b}\right) \subset I^{a-1, b-1}$. Therefore, $N_{j} \in I^{-1,-1} \mathfrak{g} \subset$ $\mathfrak{b}^{-1}$. Setting

$$
\exp \left(\sum_{j=1}^{r} z_{j} N_{j}\right)=\exp (\mathbf{z} \cdot N)
$$

we can write

$$
\exp (\mathbf{z} \cdot N) \cdot \exp \Gamma(\mathbf{s})=\exp X(\mathbf{z})
$$

for some holomorphic, $\mathfrak{b}$-valued function $X$ on $\mathcal{H}^{r}$. By construction, the period mapping is now expressed as

$$
\Phi(\mathbf{z})=\exp X(\mathbf{z}) \cdot F
$$


The horizontality of $\Phi,(3.1)$ takes the form

$$
\exp (-X) \cdot \partial \exp X \in F^{-1} \mathfrak{g}=\bigoplus_{a \geq-1} I^{a, b} \mathfrak{g}
$$

The logarithmic derivative of the $\mathfrak{b}$-valued function $X(\mathbf{z})$ must remain in $\mathfrak{b}$; therefore,

If we decompose

$$
\exp (-X) \cdot \partial \exp X \in \mathfrak{b} \cap F^{-1} \mathfrak{g}=\mathfrak{b}^{-1}
$$

$$
X=X_{-1}+X_{-2}+\cdots \quad ; \quad X_{r} \in \mathfrak{b}^{r}
$$

it follows that

$$
\exp (-X) \cdot \partial \exp X=\partial \exp X_{-1}
$$

On the other hand, we obtain from (3.3)

$$
\exp (-X) \cdot \partial \exp X=\sum_{j} e^{-\operatorname{ad}(\Gamma)} N_{j} \otimes d z_{j}+\exp (-\Gamma) \cdot \partial \exp \Gamma
$$

which, because of (3.5), implies that

$$
e^{-\operatorname{ad}(\Gamma)} N_{j}+2 \pi i s_{j} \exp (-\Gamma) \frac{\partial \exp \Gamma}{\partial s_{j}} \in \mathfrak{b}^{-1} .
$$

Setting $s_{j}=0$ and noting that $e^{-\operatorname{ad}(\Gamma)} N_{j} \equiv N_{j}\left(\bmod \mathfrak{b}^{-2}+\mathfrak{b}^{-3}+\cdots\right),(1.15)$ yields the following identity which will play an important role in our inductive step:

$$
\left[N_{j}, \Gamma(\mathbf{s})\right]=0 \text { whenever } s_{j}=0 .
$$

The differential equation (3.6) encapsulates the properties of the holomorphic function $\Gamma(\mathbf{s})$, required for $\Phi(\mathbf{z})$, defined as in (3.4), to be a period map. In fact, we have (see $[\mathbf{1 0},(2.8)])$ :

THEOREM 3.4. Let $\left\{N_{1}, \ldots, N_{r} ; F\right\}$ be a nilpotent orbit and suppose $\Gamma: \Delta^{r} \rightarrow \mathfrak{b}$ is a holomorphic map satisfying (3.4). Then, the map $\Phi(\mathbf{z})=\exp (\mathbf{z} \cdot N) \cdot \exp \Gamma(\mathbf{s}) \cdot F$ is (the lifting of) a period mapping.

Because of (3.4) we can now describe $\widetilde{\Sigma}$ as

$$
\widetilde{\Sigma}=\left\{\mathbf{z} \in \mathcal{H}^{r}: \exp (-X(\mathbf{z})) \cdot v \in F^{p}\right\}
$$

Given that the origin is in the closure of $\Sigma$ and that the filtration $F$ has been chosen as in Theorem 3.3, one can also write

$$
\widetilde{\Sigma}=\left\{\mathbf{z} \in \mathcal{H}^{r}: \exp (-X(\mathbf{z})) \cdot v=v\right\}
$$

Indeed, $v \in F^{p} \cap \bar{F}^{p} \cap W_{0}$ which, by definition of the bigrading (4.1), is contained in $I^{p, p}$. Since $X(\mathbf{z}) \in \mathfrak{b}$,

$$
\exp (X(\mathbf{z})) \cdot v \equiv v \quad\left(\bmod \bigoplus_{a<p} I^{a, b}\right)
$$

Therefore, $\exp (-X(\mathbf{z})) \cdot v \in F^{p}$ if and only if $\exp X(\mathbf{z}) \cdot v=v$. We can then conclude, since $X(\mathbf{z})$ is nilpotent:

$$
\widetilde{\Sigma}=\left\{\mathbf{z} \in \mathcal{H}^{r}: \exp X(\mathbf{z}) \cdot v=v\right\}=\left\{\mathbf{z} \in \mathcal{H}^{r}: X(\mathbf{z}) \cdot v=0\right\}
$$


Since $v \in I^{p, p}, X(\mathbf{z}) \cdot v=0 \Leftrightarrow X_{-j}(\mathbf{z}) \cdot v=0 \forall j$. Therefore

$$
\widetilde{\Sigma} \subset \widetilde{\Sigma}_{-1}:=\left\{\mathbf{z} \in \mathcal{H}^{r}: X_{-1}(\mathbf{z}) \cdot v=0\right\} .
$$

Setting $\Sigma_{-1}=\exp \left(2 \pi i \widetilde{\Sigma}_{-1}\right)$, we have a diagram of inclusions and projections:

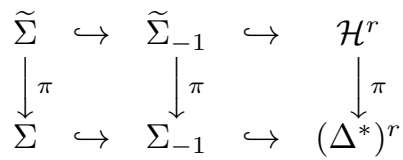

We emphasize that $\widetilde{\Sigma}$ need not be equal to $\pi^{-1}(\Sigma)$, and similarly for $\widetilde{\Sigma}_{-1}$.

LEMMA 3.5. The closure of $\Sigma_{-1}$ is an analytic subvariety of $\Delta^{r}$.

PROOF. $\Sigma_{-1}$ is defined as the projection of the variety:

$$
\widetilde{\Sigma}_{-1}=\left\{\mathbf{z} \in \mathcal{H}^{r}: X_{-1}(\mathbf{z}) \cdot v=0\right\}
$$

and

$$
X_{-1}(\mathbf{z})=\sum_{j} z_{j} N_{j}+\Gamma_{-1}(\mathbf{s})
$$

Since $v \in V_{\mathbb{Q}}$ and $N_{j} \in \operatorname{End}\left(V_{\mathbb{Q}}\right)$, we may choose coordinates $w_{j}=\sum m_{i j} z_{i}$, $m_{i j} \in \mathbb{Z}$, and a basis of $V_{\mathbb{Q}}$ so that, in these new coordinates and relative to this basis, $\widetilde{\Sigma}_{-1}$ is defined by the system of equations:

$$
\begin{gathered}
w_{j}+\mu_{j}(\mathbf{s})=0(1 \leq j \leq \rho) \\
\mu_{\rho+l}(\mathbf{s})=0(l \geq 0),
\end{gathered}
$$

where $\rho=\operatorname{dim}\left(\operatorname{span}_{\mathbb{Q}}\left\{N_{1} v, \ldots, N_{r} v\right\}\right)$ and the functions $\mu_{j}(\mathbf{s})$ are holomorphic in $\Delta^{r}$.

Let

$$
t_{j}=e^{2 \pi i w_{j}}=\prod_{i} s_{i}^{m_{i j}} .
$$

Then, $\Sigma_{-1}$ is defined by the system of equations in $\left(\Delta^{*}\right)^{r}$,

$$
\begin{gathered}
t_{j}(\mathbf{s}) e^{2 \pi i \mu_{j}(\mathbf{s})}=1(1 \leq j \leq \rho), \\
\mu_{\rho+l}(\mathbf{s})=0(l \geq 0) .
\end{gathered}
$$

From this, it is clear that the closure of $\Sigma_{-1}$ is analytic as the Lemma asserts.

THEOREM 3.6. The element $v$ is invariant under the monodromy representation of $\pi_{1}\left(\Sigma_{-1}\right)$

Proof. Given and arbitrary loop $\sigma:[0,1] \rightarrow\left(\Delta^{*}\right)^{r}$ based at $\mathbf{s}_{0}$, and its lifting $\zeta:[0,1] \rightarrow \mathcal{H}^{r}$ with $\zeta(0)=\mathbf{z}_{0}$, then

$$
\mu([\sigma])=\exp \left(\sum_{j} m_{j} N_{j}\right)
$$

where $\left(m_{1}, \ldots, m_{r}\right)=\zeta(1)-\zeta(0) \in \mathbb{Z}^{r}$. If $\sigma$ lies in $\Sigma_{-1}$, then for each $t \in[0,1]$, we can write

$$
\zeta(t)=\zeta^{\prime}(t)+q(t)
$$

with $\zeta^{\prime}(t) \in \widetilde{\Sigma}_{-1}$ and $q(t) \in \mathbb{Z}^{r}$. Since $\Gamma_{1}(0)=0$,

$$
X_{-1}(\zeta(t))=X_{-1}\left(\zeta^{\prime}(t)\right)+\sum_{j} q_{j} N_{j}
$$


and therefore

$$
X_{-1}(\zeta(t)) \cdot v=\sum_{j} q_{j} N_{j} \cdot v
$$

since $X_{-1}\left(\zeta^{\prime}(t)\right) \cdot v=0$ by definition of $\widetilde{\Sigma}_{-1}$. By continuity, and since the right-hand side of this equation lies in $V_{\mathbb{Q}}, X_{-1}(\zeta(t)) \cdot v$ must be constant. Consequently,

$$
\sum_{j} m_{j} N_{j} \cdot v=X_{-1}(\zeta(1)) \cdot v-X_{-1}(\zeta(0)) \cdot v=0
$$

Let $\bar{\Sigma}$ (respectively, $\bar{\Sigma}_{-1}$ ) denote the closure of the analytic variety $\Sigma$ (respectively, $\left.\Sigma_{-1}\right)$. Theorem 3.6 together with the following will complete the proof of Theorem 3.2.

LEMMA 3.7. $\bar{\Sigma}$ is an analytic subvariety of $\Delta^{r}$. In fact, $\bar{\Sigma}$ is a union of irreducible components of $\bar{\Sigma}_{-1}$.

Proof. The variety $\Sigma$ is defined as $\pi(\tilde{\Sigma})$, where $\tilde{\Sigma} \subset \mathcal{H}^{r}$ is defined by the equation

$$
\exp X(\mathbf{z}) \cdot v=v
$$

This equation may be interpreted as follows: Since, by assumption, the local monodromy transformations are unipotent, the vector bundle $\mathcal{V} \rightarrow\left(\Delta^{*}\right)^{r}$ has a distinguished extension $\hat{\mathcal{V}} \rightarrow \Delta^{r}$, the canonical extension [14]. A trivialization of $\hat{\mathcal{V}}$ is given by those sections of $\mathcal{V}$ corresponding to functions $\mathcal{H}^{r} \rightarrow V$ of the form

$$
\mathbf{z} \rightarrow(\exp (\mathbf{z} \cdot N)) \cdot u
$$

for $u \in V$. Consequently, the function $\mathbf{z} \rightarrow \exp X(\mathbf{z}) \cdot v$ defines a (single-valued) holomorphic section $\hat{\nu}(\mathbf{s})$ of $\hat{\mathcal{V}}$. In this setting, $\Sigma$ may be described as the locus of points $\mathbf{s} \in\left(\Delta^{*}\right)^{r}$ at which $\hat{\nu}(\mathbf{s})$ agrees with some determination of the multivalued flat section $v(\mathbf{s})$.

On the other hand, the identity (3.5) and the fact that, by definition, $X_{-1}(\mathbf{z})$. $v=0$ for $\mathbf{z} \in \tilde{\Sigma}_{-1}$, imply that $\hat{\nu}(\mathbf{s})$ defines on $\Sigma_{-1}^{*}$, the set of smooth points of $\Sigma_{-1}$, a flat, holomorphic section of the restriction of $\mathcal{V}$ to $\Sigma_{-1}^{*}$.

Suppose now that $Y$ is an irreducible component of $\bar{\Sigma}_{-1}$. Both $Y^{*}$, the subset of smooth points of $Y$, and $Y^{*} \cap\left(\Delta^{*}\right)^{r}$ are connected. If $\mathbf{s}_{0} \in Y^{*} \cap \Sigma$, then at $\mathbf{s}_{0}, \hat{\nu}\left(\mathbf{s}_{0}\right)$ agrees with some determination of $v\left(\mathbf{s}_{0}\right)$, but since $\hat{\nu}(\mathbf{s})$ is flat along $Y^{*}$, it follows that $\hat{\nu}(\mathbf{s})$ will be a determination of $v(\mathbf{s})$ for all $\mathbf{s} \in Y^{*} \cap\left(\Delta^{*}\right)^{r}$. Consequently, either $Y$ does not intersect $\bar{\Sigma}$ or $Y \subset \bar{\Sigma}$.

We conclude this section with the proof of Theorem 2.2 .

Let $U_{\mathbb{Q}} \subset\left(\mathcal{V}_{\mathbb{Q}}\right)_{\mathbf{s}_{0}}=V_{\mathbb{Q}}$ be a Hodge substructure:

$$
U=U_{\mathbb{Q}} \otimes \mathbb{C}=\bigoplus_{p+q=k} U \cap H^{p, q} .
$$

We will show that the locus $\Sigma_{U}$ of points $\mathbf{s} \in S$ at which some parallel translate of $U$ remains a Hodge substructure is an algebraic subvariety of $S$.

Let $d=\operatorname{dim}_{\mathbb{C}}(U)$ and consider the exterior power $\Lambda^{d} V$. A Hodge structure of weight $k$ on $V$ defines a Hodge structure of weight $k d$ on $\Lambda^{d} V$ by: 
with

$$
\Lambda^{d} V=\bigoplus_{a+b=k d} H_{\wedge}^{a, b}
$$

$$
H_{\wedge}^{a, b}=\bigoplus_{a_{1}+\ldots+a_{d}=a}\left(H^{a_{1}, k-a_{1}} \wedge \ldots \wedge H^{a_{d}, k-a_{d}}\right)
$$

Let $F_{\wedge}$ denote the corresponding Hodge filtration. We note that $k d$ is always even, because if $k$ is odd, then $d$ is necessarily even.

If a bilinear form $Q$ polarizes the Hodge structure on $V$, then the corresponding Hodge structure on $\Lambda^{d} V$ is polarized by the bilinear form $Q^{\wedge}$ defined by

$$
Q^{\wedge}\left(u_{1} \wedge \ldots \wedge u_{d}, v_{1} \wedge \ldots \wedge v_{d}\right)=\operatorname{det}\left(Q\left(u_{i}, v_{j}\right)\right)
$$

We consider then the polarizable variation of Hodge structure defined by $\left(\Lambda^{d} \mathcal{V}_{\mathbb{Z}}, \mathcal{F}_{\wedge}\right)$.

The one-dimensional subspace $\Lambda^{d} U$ is defined over $\mathbb{Q}$ and, at the point $\mathbf{s}_{0} \in S$, it is contained in $H_{\wedge}^{p, p}, p=k d / 2$. Thus, $\Sigma_{U} \subset \Sigma_{U}^{\wedge}$, where $\Sigma_{U}^{\wedge}$ is the locus where some parallel translate of $\Lambda^{d} U$ remains of type $(p, p)$. By Theorem (1.3), $\Sigma_{U}^{\wedge}$ is an algebraic subvariety of $S$.

We assume now that $\mathbf{s}_{0}$ is a smooth point in $\Sigma_{U}^{\wedge}$ and let $\mathcal{N}$ be a simply connected neighborhood of $\mathbf{s}_{0}$. Let $\tilde{\Sigma}_{U} \subset \mathcal{N}$ be the locus of points $\mathbf{s} \in \mathcal{N}$ such that the parallel translation of $U$ to $\mathbf{s}$ along any path in $\mathcal{N}$ is a Hodge substructure. Clearly,

$$
\tilde{\Sigma}_{U} \subset \Sigma_{U} \cap \mathcal{N} \subset \Sigma_{U}^{\wedge} \cap \mathcal{N}
$$

$\tilde{\Sigma}_{U}$ is an analytic subvariety of $\mathcal{N}$. In fact, making $\mathcal{N}$ smaller if necessary, $\tilde{\Sigma}_{U}$ is given by

$$
\tilde{\Sigma}_{U}=\left\{\mathbf{s} \in \mathcal{N}: \operatorname{dim}\left(F^{p}(\mathbf{s}) \cap U\right)=\operatorname{dim}\left(F^{p}\left(\mathbf{s}_{0}\right) \cap U\right)\right\}
$$

Assume now that $\mathbf{s}_{0}$ is a smooth point of $\tilde{\Sigma}_{U}$ as well and let

$$
\xi: T_{\mathbf{s}_{0}}(S) \longrightarrow \bigoplus_{a} \operatorname{Hom}\left(H_{\mathbf{s}_{0}}^{a, k-a}, H_{\mathbf{s}_{0}}^{a-1, k-a+1}\right)
$$

be the differential, at $s_{0}$, of the period map of the variation. The tangent space to $\tilde{\Sigma}_{U}$ at $\mathbf{s}_{0}$ is then given by

$$
T_{\mathbf{s}_{0}}\left(\tilde{\Sigma}_{U}\right)=\left\{X \in T_{\mathbf{s}_{0}}(S): \xi(X)\left(H_{\mathbf{s}_{0}}^{a, k-a} \cap U\right) \subset H_{\mathbf{s}_{0}}^{a-1, k-a+1} \cap U, \text { for all } a\right\}
$$

On the other hand, the differential of the period map associated to the variation of Hodge structure $\left(\Lambda^{d} \mathcal{V}_{\mathbb{Z}}, \mathcal{F}_{\wedge}\right)$ is given by:

$$
\xi^{\wedge}(X)=\sum_{i=1}^{d}(-1)^{i-1} I \wedge \ldots \wedge \xi(X) \wedge \ldots \wedge I
$$

and the tangent space at $\mathbf{s}_{0}$ of the $(p, p)$-locus $\Sigma_{U}^{\wedge}$ is

$$
T_{\mathbf{s}_{0}}\left(\Sigma_{U}^{\wedge}\right)=\left\{X \in T_{\mathbf{s}_{0}}(S): \xi^{\wedge}(X)\left(\Lambda^{d} U\right)=0\right\}
$$

Therefore, $T_{\mathbf{s}_{0}}\left(\tilde{\Sigma}_{U}\right)=T_{\mathbf{s}_{0}}\left(\Sigma_{U}^{\wedge}\right)$. Indeed, if $u_{1}, \ldots, u_{d}$ is a basis of $U$ compatible with the Hodge decomposition (3.8), and $X \in T_{\mathbf{s}_{0}}\left(\tilde{\Sigma}_{U}\right)$ then, for any $i=1, \ldots, d$, the product $u_{1} \wedge \ldots \wedge \xi(X) u_{i} \wedge \ldots \wedge u_{d}$ must be contained in $\Lambda^{d}(U)$, but being of type $(p-1, p+1)$, it must vanish. Conversely, if $W$ is any complement of $U$, and, given $X \in T_{\mathrm{s}_{0}}\left(\Sigma_{U}^{\wedge}\right)$ we write $\xi(X)\left(u_{i}\right)=u_{i}^{\prime}+w_{i}, u_{i}^{\prime} \in U, w_{i} \in W$, then

$$
\sum_{i}(-1)^{i-1} u_{1} \wedge \ldots \wedge\left(u_{i}^{\prime}+w_{i}\right) \wedge \ldots \wedge u_{d}=0
$$


implies that

$$
u_{1} \wedge \ldots \wedge w_{i} \wedge \ldots \wedge u_{d}=0
$$

for each $i$ and, consequently, $w_{i}=0$.

Arguing as in the proof of Lemma 3.7, we conclude that $\Sigma_{U}$ is a union of irreducible components of $\Sigma_{U}^{\wedge}$ and, therefore, algebraic.

\section{Splittings of MHS and Asymptotics of Period Maps}

The rest of the paper will be devoted to proving Theorem 3.3 for one and two variable variations. We begin by collecting the basic results on mixed Hodge structures (MHS), their real splittings, and the asymptotics of period maps that we will use in the sequel. We refer to $[\mathbf{3 4}, \mathbf{1 1}, \mathbf{1 0}, \mathbf{8}]$ for details and proofs.

Given a mixed Hodge structure (MHS) $(W, F)$ the subspaces

$$
I^{a, b}=I^{a, b}(W, F)=F^{a} \cap W_{a+b} \cap\left(\bar{F}^{b} \cap W_{a+b}+\sum_{j \geq 1} \bar{F}^{b-j} \cap W_{a+b-j-1}\right)
$$

define a bigrading of $(W, F)$ uniquely characterized by the property:

$$
I^{a, b} \equiv \overline{I^{b, a}} \quad\left(\bmod \Lambda^{a-1, b-1}\right),
$$

where

$$
\Lambda^{a, b}:=\bigoplus_{\substack{r \leq a \\ s \leq b}} I^{r, s} .
$$

We say that $(W, F)$ splits over $\mathbb{R}$ if

$$
I^{a, b}(W, F)=F^{a} \cap \bar{F}^{b} \cap W_{a+b} .
$$

We set,

$$
\begin{gathered}
L^{-1,-1}(W, F)=\left\{T \in \operatorname{End}(V): T\left(I^{a, b}\right) \subset \Lambda^{a-1, b-1}\right\} ; \\
L_{\mathbb{R}}^{-1,-1}(W, F)=L^{-1,-1}(W, F) \cap \operatorname{End}\left(V_{\mathbb{R}}\right) .
\end{gathered}
$$

Recall the following result due to Deligne [18] (see also [11]):

THEOREM 4.1. Given a MHS $(W, F)$ there exists $\delta \in L_{\mathbb{R}}^{-1,-1}(W, F)$ such that $(W, \exp (-i \delta) . F)$ is a MHS split over $\mathbb{R}$. Moreover, $\delta$ is unique, commutes with every morphism of $(W, F)$, and

$$
L^{-1,-1}(W, F)=L^{-1,-1}(W, \exp (-i \delta) \cdot F) .
$$

4.1. $S L_{2}$-orbits. If $\theta(z)=\exp (z N) F$ is a nilpotent orbit of Hodge structures of weight $2 p$, then the mixed Hodge structure $(W, F), W=W(N)[-2 p]$, is polarized by the pair $(N, Q)$ as defined in [9] (see also [11, Definition 2.26]). Indeed, the notions of polarized mixed Hodge structure (PMHS) and nilpotent orbit are equivalent [11, Corollary 3.13].

In these terms, Schmid's $S L_{2}$-orbit theorem associates to any $\operatorname{PMHS}(W, F, N, Q)$ a filtration $F_{\sigma}$ such that $\left(W, F_{\sigma}, N, Q\right)$ is a PMHS that splits over $\mathbb{R}$ - and yields the representation of $\mathfrak{s l}(2)$ that we discuss below. The filtration $F_{\sigma}$ turns out to depend only on the MHS $(W, F)$ and not on the particular $N$. Indeed, it is related to the filtration $F_{\delta}$ of Theorem 4.1 by

$$
F_{\sigma}=\exp \sigma(\delta) \cdot F_{\delta},
$$

where $\sigma(\delta)$ is a universal non-commutative polynomial in the components $\delta^{a, b}$ relative to the bigrading $I^{*, *}\left(W \mathfrak{g}, F_{\delta} \mathfrak{g}\right)$ (cf. [11, Proposition (3.28)], [17]). 
We can also write

$$
F=\exp \eta \cdot F_{\sigma}
$$

where $\eta$ is also a universal polynomial on the components of $\delta$ and, consequently, $\eta \in L^{-1,-1}(W, F) \mathfrak{g}$ as well.

Fix $(N, Q)$. Let $(W, F)$ be a MHS polarized by this pair and $\left(W, F_{\sigma}\right)$ as above. The bigrading (4.1) is given by

$$
I_{\sigma}^{a, b}=F_{\sigma}^{a} \cap \overline{F_{\sigma}^{b}} \cap W_{a+b} .
$$

Let $Y_{\sigma} \in \mathfrak{g}_{\mathbb{R}}=\mathfrak{g} \cap \operatorname{End}(V, \mathbb{R})$ be defined by:

$$
Y_{\sigma} v=(a+b-2 p) v, \quad \text { if } v \in I_{\sigma}^{a, b} .
$$

Then, the eigenspaces of $Y_{\sigma}$ :

$$
E_{\ell}\left(Y_{\sigma}\right)=\bigoplus_{a+b=\ell} I_{\sigma}^{a, b}
$$

define a real grading of $W$. Similarly, we define $I_{\delta}^{a, b}, Y_{\delta}$, and $E_{\ell}\left(Y_{\delta}\right)$.

Since $N$ is a $(-1,-1)$-morphism of $\left(W, F_{\sigma}\right)$ we have

$$
\left[Y_{\sigma}, N\right]=-2 N \text {. }
$$

In fact, $\left\{Y_{\sigma}, N\right\}$ extends to an $\mathfrak{s l}_{2}$-triple which defines a Lie algebra homomorphism:

$$
\rho_{\sigma}: \mathfrak{s l}_{2}(\mathbb{R}) \rightarrow \mathfrak{g}_{\mathbb{R}} .
$$

A similar construction could be carried out for the splitting $I_{\delta}^{a, b}$ leading to a homomorphism

$$
\rho_{\delta}: \mathfrak{s l}_{2}(\mathbb{R}) \rightarrow \mathfrak{g}_{\mathbb{R}}
$$

But it is $\rho_{\sigma}$ that generalizes well to the multivariable case.

We shall describe the construction in the case $r=2, s=0$. Let

$$
\theta\left(z_{1}, z_{2}\right)=\exp \left(z_{1} N_{1}+z_{2} N_{2}\right) F
$$

be a nilpotent orbit. We will denote by

- $W^{(2)}:=W\left(N_{1}+N_{2}\right)[-2 p]$,

- $W^{(1)}:=W\left(N_{1}\right)[-2 p]$.

- $F_{\sigma}^{(2)}$ the splitting associated with the PMHS $\left(W^{(2)}, F\right)$.

Since $\left(W^{(2)}, F_{\sigma}^{(2)}\right)$ splits over $\mathbb{R}$ and the action of $N_{2}$ on $W^{(1)}$ agrees with the action of $N_{1}+N_{2}$, it follows that $\left(W^{(1)}, \exp \left(i N_{2}\right) F_{\sigma}^{(2)}\right)$ is a polarized MHS. We denote by

- $F_{\sigma}^{(1)}$ the splitting associated with the PMHS $\left(W^{(1)}, \exp \left(i N_{2}\right) F_{\sigma}^{(2)}\right)$.

- $Y_{\sigma}^{(2)}$ and $Y_{\sigma}^{(1)}$, the semisimple transformations (4.4).

We recall some basic results from $[\mathbf{1 1}]$ (see also [10]):

THEOREM 4.2. The semisimple elements $Y_{\sigma}^{(2)}$ and $Y_{\sigma}^{(1)}$ commute and, consequently, define a real bigrading

$$
V=\bigoplus_{\left(\ell_{1}, \ell_{2}\right) \in \mathbb{Z}^{2}} V_{\ell_{1}, \ell_{2}}
$$

such that $Y_{\sigma}^{(j)}$ acts as multiplication by $\ell_{j}$ on $V_{\ell_{1}, \ell_{2}}$. Let $\hat{N}_{2}$ be the component of $N_{2}$ in the zero-eigenspace of $\operatorname{ad}\left(Y_{\sigma}^{(1)}\right)$. Then

$$
W\left(N_{1}+N_{2}\right)=W\left(N_{1}+\hat{N}_{2}\right)
$$


and the pairs $\left\{Y_{\sigma}^{(1)}, N_{1}\right\}$ and $\left\{Y_{\sigma}^{(2)}-Y_{\sigma}^{(1)}, \hat{N}_{2}\right\}$ determine commuting $\mathfrak{s l}_{2}$-triples. Moreover:

$$
\exp \left(i N_{1}\right) \cdot F_{\sigma}^{(1)}=\exp \left(i\left(N_{1}+\hat{N}_{2}\right)\right) \cdot F_{\sigma}^{(2)} .
$$

This filtration, which lies in $D$, will be denoted by $F_{\#}$.

Note that the last assertion of Theorem 4.2 implies that

$$
\left(W^{(1)}, F_{\#}\right) \text { and }\left(W^{(2)}, F_{\#}\right) \text { are PMHS. }
$$

We remark also that the construction above depends on the ordering of the variables $z_{1}, z_{2}$ and that Theorem 4.2 fails if we replace the splittings $F_{\sigma}$ by the splitting $F_{\delta}$.

Lemma 4.3. With notation as above, let $\theta(z)=\exp (z N) F$ be a nilpotent orbit. Then

a) $F^{p} \cap V_{\mathbb{R}} \cap W_{0}(N) \subset E_{0}\left(Y_{\delta}^{(2)}\right) \cap \operatorname{ker}(\delta)$.

b) $F^{p} \cap V_{\mathbb{R}} \cap W_{0}(N) \subset E_{0}\left(Y_{\sigma}^{(2)}\right) \cap \operatorname{ker}(\sigma)$.

Proof. Suppose $v$ is in the subspace defined by the left-hand side of a). Then, $v \in F^{p} \cap W_{2 p} \cap V_{\mathbb{R}}$ and, therefore,

$$
v \in I_{(W, F)}^{p, p}=\exp (i \delta) I_{\left(W, F_{\delta}\right)}^{p, p} .
$$

Therefore, if we write $v=e^{i \delta} f, f \in I_{\left(W, F_{\delta}\right)}^{p, p}$, we have $e^{i \delta} f=e^{-i \delta} \bar{f}$ or, equivalently,

$$
e^{2 i \delta} f=\bar{f} \text {. }
$$

But, since $f \in I_{\left(W, F_{\delta}\right)}^{p, p}$ and

$$
\delta \in L^{-1,-1}\left(W, F_{\delta}\right),
$$

this is possible only if $\delta f=0$ and $v=f$. The second statement follows from the fact that $F_{\sigma}=\exp (\sigma) F_{\delta}$, where $\sigma$ is a polynomial on the components of $\delta$. This means that an element $v$ on the left-hand side of b) is annihilated by $\sigma$ as well.

This result may be generalized to the bivariate case:

Lemma 4.4. With notation as above, let $\theta\left(z_{1}, z_{2}\right)$ be a nilpotent orbit. Then:

a) $F^{p} \cap V_{\mathbb{R}} \cap \operatorname{ker}\left(N_{1}\right) \cap \operatorname{ker}\left(N_{2}\right) \subset E_{0}\left(Y_{\sigma}^{(2)}\right) \cap E_{0}\left(Y_{\sigma}^{(1)}\right)$.

b) $F^{p} \cap V_{\mathbb{R}} \cap \operatorname{ker}\left(N_{1}\right) \cap \operatorname{ker}\left(N_{2}\right) \subset E_{0}\left(Y_{\delta}^{(2)}\right) \cap E_{0}\left(Y_{\delta}^{(1)}\right)$.

c) $F_{\#}^{p} \cap W_{0}\left(N_{1}\right) \cap V_{\mathbb{R}} \subset E_{0}\left(Y_{\sigma}^{(1)}\right) \cap \operatorname{ker}\left(N_{1}\right)$.

Proof. To prove a) we first apply b) in Lemma 4.3 to the nilpotent orbit $\exp \left(z\left(N_{1}+N_{2}\right)\right) F$ to deduce that

$$
F^{p} \cap V_{\mathbb{R}} \cap \operatorname{ker}\left(N_{1}\right) \cap \operatorname{ker}\left(N_{2}\right) \subset\left(F_{\sigma}^{(2)}\right)^{p} .
$$

Hence, if $v$ is in the left-hand side of a) we have

$$
v \in\left(\exp \left(i N_{2}\right) F_{\sigma}^{(2)}\right)^{p} \cap W_{0}\left(N_{1}\right) \cap V_{\mathbb{R}} .
$$

Applying again b) in Lemma 4.3 but this time to the nilpotent orbit

$$
\exp \left(z N_{1}\right)\left(\exp \left(i N_{2}\right) F_{\sigma}^{(2)}\right)
$$

we obtain the statement. The second assertion follows from a) by the relationship between the splittings $Y_{\sigma}^{(1)}$ and $Y_{\delta}^{(1)}$.

Since $F_{\#}=\exp \left(i N_{1}\right) F_{\sigma}^{(1)}$ and $N_{1} \in L^{-1,-1}\left(W^{(1)}, F_{\sigma}^{(1)}\right)$ it follows from the uniqueness in Theorem 4.1 that $N_{1}$ plays the role of $\delta$ for this MHS. Hence e) 
follows from Lemma 4.3 with $F_{\#}, W\left(N_{1}\right), F_{\sigma}^{(1)}, N_{1}$ in place of $F, W^{(2)}, F_{\delta}, \delta$, respectively.

4.2. Asymptotics of a Period Map. Recall from Theorem 3.4 that if

$$
\left\{N_{1}, \ldots, N_{r} ; F\right\}
$$

is a nilpotent orbit and $\Gamma: \Delta^{r} \rightarrow \mathfrak{b}$ is a holomorphic map satisfying (3.4), then the map

$$
\Phi(\mathbf{z})=\exp (\mathbf{z} \cdot N) \cdot \exp \Gamma(\mathbf{s}) \cdot F, \quad s_{j}=e^{2 \pi i z_{j}}
$$

is a holomorphic horizontal map

$$
\Phi: \mathcal{U}_{\alpha}^{r}:=\left\{\mathbf{z} \in \mathcal{U}^{r}: \operatorname{Im}\left(z_{j}\right)>\alpha\right\} \rightarrow D,
$$

for some $\alpha$ sufficiently large. We point out that this statement holds for $N_{j} \in \mathfrak{g}_{\mathbb{R}}$, not necessarily defined over $\mathbb{Q}$. We will refer to $\left\{N_{1}, \ldots, N_{r} ; F ; \Gamma\right\}$ as period mapping data.

Suppose now that $(N ; F ; \Gamma)$ is as above. Let $W=W(N)[-2 p]$ and let $F_{\sigma}, Y_{\sigma}$, be as before. Set

$$
e(y)=\exp \left(\frac{1}{2} \log y Y_{\sigma}\right) \in G
$$

Since

$$
F_{\sigma}^{r}=\bigoplus_{a \geq r} I_{\sigma}^{a, b}
$$

and $I_{\sigma}^{a, b}$ is contained in an eigenspace of $Y_{\sigma}$, the linear transformations $e(y)$ preserve the filtration $F_{\sigma}$.

LEMMA 4.5. With notation as above, if $x=\operatorname{Re}(z)$ remains bounded then

$$
\lim _{y \rightarrow \infty} e(y) \Phi(z)=F_{\#}=\exp (i N) F_{\sigma} .
$$

Proof. We can write:

$$
\begin{aligned}
\Phi(z) & =\exp (z N) \cdot \psi(s) \\
& =\exp (x N) \cdot \exp (i y N) \cdot \exp (\Gamma(s)) \cdot F \\
& =\exp (x N) \cdot e^{-1}(y) \cdot \exp (i N) \cdot e(y) \cdot \exp (\Gamma(s)) \cdot e^{\eta} \cdot F_{\sigma},
\end{aligned}
$$

where $\eta \in L^{-1,-1} \mathfrak{g}$ is such that $F=e^{\eta} F_{\sigma}$. Therefore

$$
\begin{aligned}
e(y) \cdot \Phi(z) & =e(y) \cdot \exp (x N) \cdot e^{-1}(y) \cdot e^{i N} \cdot e(y) \cdot \exp (\Gamma(s)) \cdot e^{\eta} \cdot F_{\sigma} \\
& =\exp ((x / y) N) \cdot e^{i N} \cdot e(y) \cdot \exp (\Gamma(s)) \cdot e^{\eta} \cdot F_{\sigma} \\
& =\exp ((x / y) N) \cdot e^{i N} \cdot e(y) \cdot \exp (\Gamma(s)) \cdot e^{\eta} \cdot e^{-1}(y) \cdot F_{\sigma}
\end{aligned}
$$

where the last equality follows from the fact that $e(y) \cdot F_{\sigma}=F_{\sigma}$. The statement now follows from the fact that both $\Gamma(s)$ and $\eta$ live in the sum of eigenspaces of $\operatorname{ad}\left(Y_{\sigma}\right)$ corresponding to strictly negative eigenvalues.

We will need a generalization of Lemma 4.5 to the multivariable case. Again, to simplify the exposition, we will restrict ourselves the two-variable case. We continue with the same notation as above. 
Let $\left(N_{1}, N_{2} ; F ; \Gamma\right)$ be period mapping data and $\Phi: \mathcal{U}_{\alpha}^{2} \rightarrow D$ the associated map. Let $W^{(2)}, W^{(1)}, F_{\sigma}^{(2)}, F_{\sigma}^{(1)}, Y_{\sigma}^{(2)}, Y_{\sigma}^{(1)}, \hat{N}_{2}$, as before. We set $t_{2}=y_{2}, t_{1}=y_{1} / y_{2}$, and

$$
e(\mathbf{t}):=\exp \left(\frac{1}{2} \log t_{1} Y_{\sigma}^{(1)}\right) \exp \left(\frac{1}{2} \log t_{2} Y_{\sigma}^{(2)}\right)
$$

The following result is contained in Lemma (4.5) and Remark (4.6) of [10]. We give a sketch and refer to $[\mathbf{1 0}]$ for details.

Lemma 4.6. Suppose $x_{1}=\operatorname{Re}\left(z_{1}\right)$ and $x_{2}=\operatorname{Re}\left(z_{2}\right)$ are bounded. Then, if $t_{1}$ remains bounded away from zero and $t_{2} \rightarrow \infty$,

a) $e(\mathbf{t}) \cdot \exp \left(x_{1} N_{1}+x_{2} N_{2}\right) \cdot e(\mathbf{t})^{-1}$ converges to the identity and

$$
\left\|e(\mathbf{t}) \cdot \exp \left(x_{1} N_{1}+x_{2} N_{2}\right) \cdot e(\mathbf{t})^{-1}-1\right\|=O\left(t_{2}^{-1 / 2}\right)
$$

b) $e(\mathbf{t}) \cdot \exp \left(y_{1} N_{1}+y_{2} N_{2}\right) \cdot e(\mathbf{t})^{-1}$ converges to $\exp \left(i\left(N_{1}+\hat{N}_{2}\right)\right)$ and

$$
\left\|e(\mathbf{t}) \cdot \exp \left(x_{1} N_{1}+x_{2} N_{2}\right) \cdot e(\mathbf{t})^{-1}-\exp \left(i\left(N_{1}+\hat{N}_{2}\right)\right)\right\|=O\left(t_{2}^{-1 / 2}\right)
$$

c) $\left\|e(\mathbf{t}) \gamma(\mathbf{s}) e(\mathbf{t})^{-1}-1\right\|=O\left(e^{-\alpha t_{2}}\right)$, for a positive constant $\alpha$.

d) $e(\mathbf{t}) \Phi\left(z_{1}, z_{2}\right) e(\mathbf{t})^{-1} \rightarrow F_{\#}$.

Proof. Since $N_{1}$ and $N_{2}$ are $(-1,-1)$-morphisms of $\left(W^{(2)}, F_{\sigma}^{(2)}\right)$, they lie in the sum of eigenspaces of $\operatorname{ad}\left(Y_{\sigma}^{(2)}\right)$ with eigenvalues less than or equal to -2 . On the other hand, $N_{1}$ is also a $(-1,-1)$-morphism of $\left(W^{(1)}, F_{\sigma}^{(1)}\right)$, and, since $N_{2}$ commutes with $N_{1}$ it preserves $W\left(N_{1}\right)$. Consequently $N_{2}$ lies in the sum of eigenspaces of $\operatorname{ad}\left(Y_{\sigma}^{(1)}\right)$ with non-positive eigenvalues. Thus, a) follows. A similar argument yields b).

In order to prove $\mathrm{c})$, we recall that $\gamma(\mathbf{s})=\exp (\Gamma(\mathbf{s}))$ and decompose

$$
\Gamma\left(s_{1}, s_{2}\right)=\sum_{\ell_{1}, \ell_{2}} \Gamma_{\ell_{1}, \ell_{2}}\left(s_{1}, s_{2}\right) .
$$

By construction we must have that for $\ell_{2} \geq 0$,

$$
\Gamma_{\ell_{1}, \ell_{2}}(\mathbf{s})=0 \text {. }
$$

Suppose now that $\ell_{1}>0$, and consider a component $\Gamma_{\ell_{1}, \ell_{2}}(\mathbf{s})$. By (3.7)

$$
\left[\Gamma_{\ell_{1}, \ell_{2}}\left(0, s_{2}\right), N_{1}\right]=0 .
$$

Therefore for $\ell_{1}>0, \Gamma_{\ell_{1}, \ell_{2}}\left(0, s_{2}\right)=0$. Hence, for $\ell_{1}>0$

$$
\left\|\operatorname{Ad}\left(\exp \left(\frac{1}{2} \log t_{1} Y_{\sigma}^{(1)}\right)\right) \Gamma_{\ell_{1}, \ell_{2}}\right\|=O\left(t_{1}^{\ell_{1} / 2} e^{-\beta y_{1}}\right)
$$

which converges to zero as $y_{2} \rightarrow \infty$.

The last statement follows from a), b), and c) as in the proof of Lemma 4.5.

\section{The Local Statement: One variable case.}

In this section we will prove Theorem 3.3 in the case of one-variable period mappings. The argument follows roughly the lines of [15] though we have placed it in the context needed for the general argument.

We will prove Theorem 3.3 by contradiction. Indeed, its assertions wil follow from the following: 
THEOREM 5.1. Let $\left(N_{1}, \ldots, N_{r} ; F ; \Gamma\right)$ be period mapping data and $K>0$. Suppose there exist sequences $u(n) \in V_{\mathbb{Z}}$ and $\mathbf{z}(n)=\mathbf{x}(n)+i \mathbf{y}(n) \in \mathcal{U}^{r}$ such that:

a) $Q(u(n), u(n)) \leq K$.

b) $\lim _{n \rightarrow \infty} x_{i}(n)$ exists for all $i=1, \ldots, r$.

c) $\lim _{n \rightarrow \infty} y_{i}(n)=\infty$ for all $i=1, \ldots, r$.

d) $u(n) \in \Phi^{p}(\mathbf{z}(n))$.

Then, for $n$ sufficiently large, $u(n)$ takes values in a finite set

$$
\left\{v_{1}, \ldots, v_{m}\right\} \subset W_{0}\left(N_{1}+\cdots+N_{r}\right) \cap F^{p},
$$

for some limiting Hodge filtration $F \in \check{D}$.

Note that if $u(n)$ satisfies the assumptions then any subsequence does as well. In the arguments that follow, we will freely pass to subsequences while keeping the original notation.

We will prove this theorem in the cases $r=1$ and $r=2$, where the arguments are representative of those used in the general case but become more transparent.

The inductive nature of the proof makes it necessary to prove a different version of Theorem 5.1. We do this in the one-variable case. In what follows, the expression $\| \bullet$ means the Hodge norm at the point $F_{\#} \in D$.

Proposition 5.2. Let $(N ; F ; \Gamma)$ define a period map. Let $u(n), z(n)$ be sequences such that:

a) $u(n) \in V_{\mathbb{Z}}$.

b) $z(n)=x(n)+i y(n) \in \mathcal{U}$ and, as $n \rightarrow \infty$,

$$
x(n) \rightarrow x_{0} \in \mathbb{R}, \quad y(n) \rightarrow \infty .
$$

c) $u(n)=f(n)+g(n)$, where $f(n) \in \Phi^{p}(z(n))$ and

$$
\|g(n)\| \sim e^{-\alpha y(n)}\|u(n)\|
$$

as $n \rightarrow \infty$ for some positive constant $\alpha$.

d) $\|e(n) u(n)\|$ is bounded, where $e(n)=e(y(n))$.

Then, there exists a finite collection

$$
\left\{v_{1}, \ldots, v_{m}\right\} \subset W_{0}(N) \cap F^{p},
$$

such that, for $n$ sufficiently large, $u(n)=v_{j}$ for some $j$. Moreover, each $v_{j}$ is fixed by the $\mathfrak{s l}_{2}$-representation $\rho_{\sigma}$.

REMARK 5.3. If assumption iii) in Proposition 5.2 is satisfied we say

$$
u(n) \sim_{y(n)} \Phi^{p}(z(n))
$$

Before proving Proposition 5.2 let us observe that it implies Theorem 5.1 in the case $r=1$. Indeed, it suffices to check that if $u(n), z(n)$ satisfy the conditions in Theorem 5.1, then d) in Proposition 5.2 is satisfied as well. Since

$$
u(n) \in V_{\mathbb{R}} \cap \Phi^{p}(z(n))
$$

and $e(y(n)) \in G_{\mathbb{R}}$, we have

$$
\begin{aligned}
Q(u(n), u(n)) & =\|u(n)\|_{\Phi(z(n))}^{2} \\
& =\|e(y(n)) u(n)\|_{e(y(n)) \Phi(z(n))}^{2} .
\end{aligned}
$$




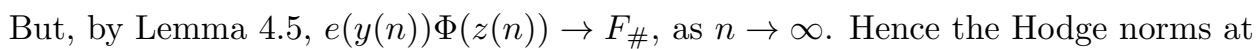
the points $e(y(n)) \Phi(z(n))$ and $F_{\#}$ are uniformly mutually bounded. The statement now follows from the assumption that $Q(u(n), u(n)) \leq K$.

\section{Proof of Proposition 5.2}

We note first of all that the statement and conclusion of the Proposition remain unchanged if we replace the period map $\Phi$ by the associated nilpotent orbit $\theta(z)=$ $\exp (z N) \cdot F$. Thus, in what follows we will assume that $\Gamma(s)=0$. Let $u(n)=$ $\sum_{\ell} u(n)_{\ell}$ be the decomposition of $u(n)$ in eigenspaces of $Y_{\sigma}$ and let $\ell_{0}$ be the largest $\ell$ such that $u(n)_{\ell_{0}} \neq 0$ for infinitely many $n$ 's. Then, for those $n$ :

$$
\|e(n) u(n)\| \sim y(n)^{\ell_{0} / 2}\left\|u(n)_{\ell_{0}}\right\|
$$

and it follows from the assumptions that $\ell_{0} \leq 0$. Hence, for $n$ sufficiently large

$$
u(n) \in W_{0}(N)
$$

Moreover, for those $n$ 's such that $u(n)_{0} \neq 0$, we have from $(5.2)$ that $\left\|u(n)_{0}\right\|$ is bounded. Hence, since $u(n) \in V_{\mathbb{Z}}$ we deduce that the elements $u(n)_{0}$ vary in a finite set

$$
\left\{v_{1}, \ldots, v_{m}\right\} \subset E_{0}\left(Y_{\sigma}\right) .
$$

We may assume without loss of generality that $m=1$ and set $v=v_{1}$. Hence, we may assume that $u(n)=v+w(n)$, where $w(n) \in W_{-1}(N)$.

The next step will be to show that

$$
v \in I_{\sigma}^{p, p} \cap \operatorname{ker} N \text { and } \lim _{n \rightarrow \infty} e\left(y_{n}\right) w(n)=0 .
$$

Lemma 5.4. Let $T: U \rightarrow V$ be a linear map of vector spaces. Let $L_{n}$ be a sequence of lines in $U$ converging to a line $L$ which is complementary to $\operatorname{ker} T$. Then $T\left(L_{n}\right)$ converge to $T(L)$.

ProOF. We may assume without loss of generality that $L_{n}$ is complementary to $\operatorname{ker} T$ for all $n$. Let $\tilde{L}_{n}$ be the projection to $U / \operatorname{ker} T$, clearly the lines $\tilde{L}_{n}$ converge to $\tilde{L}$. The result now follows from the injection $U / \operatorname{ker} T \rightarrow V$.

Returning to the proof of (5.5), let $L_{n}$ be the line in $H$ spanned by $e(n) u(n)$. Passing to a subsequence if necessary, we may assume that $L_{n}$ converges to a line $L$. Now, by assumption we may write

$$
e(n) u(n)=e(n) f(n)+e(n) g(n),
$$

where $f(n) \in \theta(z(n))$ and $\|g(n)\| /\|u(n)\| \rightarrow 0$ exponentially as $n \rightarrow \infty$. Since $e(n)$ grows at worst polynomially on $y(n)$, the same is true for $\|e(n) g(n)\| /\|e(n) u(n)\|$. Hence, we may assume that the lines $\mathbb{R}(e(n) u(n))$ and $\mathbb{R}(e(n) f(n))$ converge to the same line $L$. But, by Lemma 4.5, $e(n) \theta(z(n)) \rightarrow F_{\#}$ and, therefore,

$$
L \subset F_{\#}^{p} \cap W_{0}(N) \cap H_{\mathbb{R}}
$$

Hence, by Lemma 4.3,

$$
L \subset I_{\sigma}^{p, p} \cap \operatorname{ker} N \text {. }
$$

Consider now the projection $T: H \rightarrow E_{0}(Y)$. Clearly $L \cap \operatorname{ker} T=\{0\}$, in fact $\left.T\right|_{L}=i d$. Hence, it follows from Lemma 5.4 that

$$
L=T(L)=\lim _{n} T\left(L_{n}\right)=\lim _{n} \mathbb{R} \cdot v=\mathbb{R} \cdot v
$$

and, consequently, $v \in I_{0}^{p, p} \cap \operatorname{ker} N$. 
To prove the second statement choose $\lambda_{n} \in \mathbb{R}$ such that

$$
\lambda_{n} e(n) u(n)=\lambda_{n} v+\lambda_{n} e(n) w(n)
$$

converges to a non-zero element of the limiting line $L=\mathbb{R} \cdot v$. This means that $\lambda_{n}$ converges to some $\lambda \neq 0$ and

$$
0=\lim _{n \rightarrow \infty} \lambda_{n} e(n) w(n)=\lambda \lim _{n \rightarrow \infty} e(n) w(n) .
$$

We will next show that for $n$ sufficiently large, $u(n) \in \operatorname{ker} N$ or, equivalently,

$$
N(w(n))=0 .
$$

Since $e(n)$ acts polynomially on $y(n)^{-1 / 2}$, it suffices to show that

$$
\| N(e(n)) u(n))\|/\| u(n) \|=O\left(e^{-\alpha y(n)}\right) .
$$

Now,

$$
\begin{aligned}
N(e(n)) w(n)) & =N(e(n)) u(n)) \\
& =N(e(n)) f(n))+N(e(n)) g(n))
\end{aligned}
$$

with $\| N(e(n)) g(n))\|/\| u(n) \|=O\left(e^{-\beta y(n)}\right)$. But $\left.\left.\| N(e(n)) u(n)\right)\|/\| N(e(n)) g(n)\right) \|$ is bounded since, otherwise, we could assume that the lines $\mathbb{R} N(e(n)) w(n))$ and $\mathbb{R} N(e(n)) f(n))$ have the same limit $L$. But then $L \subset F_{\#}^{p-1} \cap W_{3}(N) \cap V_{\mathbb{R}}=\{0\}$. Since $\| N(e(n)) g(n)) \|=O\left(e^{-\beta y(n)}\right)$ and $e(n)$ acts at most polynomially on $y(n)$, (5.7) follows. Now, since $u(n) \in V_{\mathbb{Z}}$, they lie in a discrete set and then so do $N(w(n))$. Hence $N(w(n))=0$ for $n$ sufficiently large and we get (5.6).

Now, $u(n)=\exp (-z(n) N)(f(n)+g(n))$ and $\exp (-z(n) N) f(n) \in F$. Hence the lines $L_{n}$ spanned by $u(n)$ converge to a line

$$
L \subset W_{0}(N) \cap F^{p} \cap V_{\mathbb{R}}
$$

Now, $u(n)=\exp (-z(n) N)(f(n)+g(n))$ and $\exp (-z(n) N) f(n) \in F$. Hence, for $n$ sufficiently large $u(n)$ is bounded in the Hodge norm at a point in $D$ and, being in the lattice, may take only finitely many values which must lie in $\operatorname{ker} N \cap W_{0}(N) \cap F$. But then Lemma 4.3 implies that $L \subset E_{0}\left(Y_{\sigma}\right)$ and, consequently, $u(n)=v$ for $n$ sufficiently large. Moreover, since $v \in \operatorname{ker} N \cap \operatorname{ker}\left(Y_{\sigma}\right)$, it follows that $v$ is fixed by the $\mathfrak{s l}_{2}$-representation $\rho_{\sigma}$. This completes the proof of Proposition 5.2.

REMARK 5.5. We point out that in the arguments of this section we could have replaced the split MHS $\left(W, F_{\sigma}\right)$ by $\left(W, F_{\delta}\right)$ throughout. Indeed it is only in the multivariable setting that the splitting given by the $S L_{2}$-orbit theorem becomes essential.

\section{The Local Statement: Bivariate Case}

Let $u(n), \mathbf{z}(n)=\left(z_{1}(n), z_{2}(n)\right)$ be as in the statement of Theorem 5.1. Passing to a subsequence and exchanging the roles of $N_{1}$ and $N_{2}$ if necessary, we may assume, without loss of generality, that $y_{1}(n) / y_{2}(n)$ is bounded away from zero. We will consider separately the cases when $t_{1}(n)=y_{1}(n) / y_{2}(n)$ is bounded and unbounded.

We begin with the latter case. We may then assume $t_{1}(n) \rightarrow \infty$ as $n \rightarrow \infty$. As before, let $e(n)=e\left(t_{1}(n), t_{2}(n)\right)$. Note that we can argue as in the one-variable case to deduce that $u(n) \in \Phi^{p}(\mathbf{z}(n))$ implies that

$$
\|e(n) u(n)\| \text { is bounded. }
$$


Let $\left(\ell_{1}, \ell_{2}\right)$ be the largest pair of indices, in the lexicographic order, such that there is a subsequence, also denoted by $u(n)$ with

$$
u(n) \in W_{\ell_{1}}\left(N_{1}\right) \cap W_{\ell_{2}}\left(N_{1}+N_{2}\right)
$$

and non-trivial projections to the corresponding graded quotients.

We may assume that the lines $L_{n}=\mathbb{R} \cdot(e(n) u(n))$ converge to a line $L$ which must lie in

$$
F_{\#}^{p} \cap W_{\ell_{1}}\left(N_{1}\right) \cap V_{\mathbb{R}} .
$$

But, since $\left(W\left(N_{1}\right)[-2 p], F_{\#}^{p}\right)$ is a mixed Hodge structure, the fact that the subspace (6.2) is non-trivial implies that $\ell_{1} \geq 0$.

Suppose now that $\ell_{1}>0$. Since $e(n) u(n)$ is bounded, this means that $\ell_{2}<0$. Let $\tilde{\Phi}^{(1)}\left(z_{2}\right)$ be the projection to $\operatorname{Gr}_{\ell_{1}}^{W\left(N_{1}\right)}$ of

$$
\Phi^{(1)}\left(z_{2}\right)=\exp \left(z_{2} N_{2}\right) \gamma\left(0, s_{2}\right) F .
$$

Because of $(3.7), \gamma\left(0, s_{2}\right)$ preserves the weight filtration $W\left(N_{1}\right)$, and we can apply Theorem 3.4 to conclude that $\tilde{\Phi}^{(1)}\left(z_{2}\right)$ is a period map of Hodge structures of weight $2 p+\ell_{1}$. Its nilpotent orbit is given by $\left(\tilde{N}_{2}, \tilde{F}\right)$, where $\tilde{N}_{2}$ and $\tilde{F}$ denote the projections of $N_{2}$ and $F$ to $\operatorname{Gr}_{\ell_{1}}^{W\left(N_{1}\right)}$. Moreover, $W_{\ell}\left(\tilde{N}_{2}\right)$ is the projection to $\mathrm{Gr}_{\ell_{1}}^{W\left(N_{1}\right)}$ of $W_{\ell+\ell_{1}}\left(N_{1}+N_{2}\right)$ (cf. [9]), and the $S L_{2}$-splitting is given by the projection $\tilde{Y}_{\sigma}^{(2)}$ of $Y_{\sigma}^{(2)}$. In particular,

$$
\tilde{e}(n)[u(n)]=[e(n) u(n)] \text { is bounded in norm. }
$$

Consider now the lines $L_{n}=\mathbb{R}[e(n) u(n)]$, which we may assume convergent. The limiting line is real and lies in

$$
W_{\ell_{2}-\ell_{1}}\left(\tilde{N}_{2}\right) \cap \tilde{F}^{p} .
$$

But, since $\ell_{2}-\ell_{1}<0$, the intersection (6.4) is $\{0\}$. Hence $\ell_{1}=0$.

The same argument, with $\ell_{1}=0$ gives that $\ell_{2} \geq 0$. But if $\ell_{2}>0$ the sequences $e(n) u(n)$ would be unbounded. Hence

$$
u(n) \in W_{0}\left(N_{1}\right) \cap W_{0}\left(N_{1}+N_{2}\right) .
$$

Let $[u(n)]$ denote the projection of $u(n)$ and $\tilde{\Phi}^{(1)}\left(z_{2}\right)$ the projection of $\Phi^{(1)}\left(z_{2}\right)$ to $\operatorname{Gr}_{0}^{W\left(N_{1}\right)}$. We claim that

$$
[u(n)] \sim_{y_{1}(n)} \tilde{\Phi}^{(1)}\left(z_{2}\right) .
$$

Note first of all that $\Gamma\left(s_{1}, s_{2}\right)=\Gamma\left(0, s_{2}\right)+s_{1} B\left(s_{1}, s_{2}\right)$ with $B$ a $\mathfrak{b}$-valued holomorphic map on $\Delta^{2}$. Therefore,

$$
\gamma\left(s_{1}, s_{2}\right)=\mu\left(s_{1}, s_{2}\right) \gamma\left(0, s_{2}\right)
$$

and $\left\|\mu\left(s_{1}, s_{2}\right)-1\right\|<K e^{-2 \pi y_{1}}$. Hence

$$
\exp \left(-z_{1} N_{1}\right) \Phi\left(z_{1}, z_{2}\right)=\left(e^{z_{2} N_{2}} \mu\left(s_{1}, s_{2}\right) e^{-z_{2} N_{2}}\right) \Phi^{(1)}\left(z_{2}\right) .
$$

But, since $y_{1}(n) / y_{2}(n) \rightarrow \infty$ we have that as $n \rightarrow \infty$,

$$
\left\|e^{z_{2}(n) N_{2}} \mu(\mathbf{s}(n)) e^{-z_{2}(n) N_{2}}-1\right\|<A e^{-\alpha y_{1}(n)} .
$$

Hence

$$
\exp \left(-z_{1}(n) N_{1}\right) u(n) \sim_{y_{1}(n)} \Phi^{(1)}\left(z_{2}\right)
$$


Since, $\left[\exp \left(-z_{1}(n) N_{1}\right) u(n)\right]=[u(n)]$, it remains to check that the order of convergence is preserved by passage to $\mathrm{Gr}_{0}^{W_{1}(N)}$. This follows by noting that our assumptions guarantee that as $n \rightarrow \infty$, the ratio $\|u(n)\| /\|[u(n)]\|$ grows at worst polynomially with $y_{1}(n)$.

Given (6.3), it follows then that $[u(n)]$ satisfies the assumptions of Proposition 5.2 for the period map data: $\left(\tilde{N}_{2}, \tilde{F}, \Gamma\left(0, s_{2}\right)\right)$ in $\operatorname{Gr}_{0}^{W\left(N_{1}\right)}$. Hence, there are only finitely may $[u(n)]$ and they are fixed by the $\mathfrak{s l}_{2}$-triple defined by $\tilde{N}_{2}$ and $\tilde{Y}_{\sigma}^{(2)}$. Thus, we may assume without loss of generality that $[u(n)]$ is constant; i.e.

$$
u(n)=v+w(n)
$$

where $v \in E_{0}\left(Y_{\sigma}^{(2)}\right)$ and $w(n) \in W_{-1}\left(N_{1}\right)$. We may now mimick the argument used to prove (5.5) to deduce that

$$
v \in \operatorname{ker} N_{1} \quad \text { and } \quad \lim _{n \rightarrow \infty} e(n) w(n)=0 .
$$

As in the proof of Proposition 5.2, the next step is to show that

$$
\left\|N_{1} u(n)\right\|<\|u(n)\| e^{-\alpha y_{1}(n)},
$$

which implies that $N_{1} u(n)=0$ for $n$ sufficiently large as $N_{1} u(n)$ takes values in a discrete set. This is done exactly as in the proof of (5.7) replacing $N, e(y(n)), \Phi(z)$ by $N_{1}, \exp \left(1 / 2 \log y_{1}(n) Y_{\sigma}^{(1)}\right.$, and $\Phi^{(1)}\left(z_{2}\right)$.

Finally, since $u(n) \in \operatorname{ker} N_{1}$ we have that $u(n)$ satisfies the conditions of Proposition 5.2 for the period map data $\left(N_{2} ; \exp \left(i \lambda N_{1}\right) F ; \Gamma\left(0, s_{2}\right)\right)$ for some $\lambda \in \mathbb{R}$. This means that $u(n)$ itself varies in a finite set $\left\{v_{1}, \ldots, v_{m}\right\}$ contained in

$$
E_{0}\left(Y_{\sigma}^{(1)}\right) \cap E_{0}\left(Y_{\sigma}^{(2)}\right) \cap F^{p} .
$$

This completes the proof of Theorem 5.1 in the case $t_{1}(n)=y_{1}(n) / y_{2}(n)$ is unbounded.

Suppose now that $t_{1}(n) \rightarrow \lambda \neq 0$ as $n \rightarrow \infty$. We can then rewrite:

$$
y_{1} N_{1}+y_{2} N_{2}=y_{2}\left(\lambda N_{1}+N_{2}\right)+\left(y_{1}-\lambda y_{2}\right) N_{1} \text {. }
$$

Note that

$$
\lim _{n \rightarrow \infty} \frac{y_{2}(n)}{y_{1}(n)-\lambda y_{2}(n)}=\infty
$$

and that we may assume without loss of generality that $y_{1}(n)-y_{2}(n) \lambda>0$ for all $n$. Therefore, if

$$
\lim _{n \rightarrow \infty}\left(y_{1}(n)-\lambda y_{2}(n)\right)=\infty
$$

we may replace the period mapping data $\left(N_{1}, N_{2} ; F, \Gamma\right)$ by $\left(\lambda N_{1}+N_{2}, N_{1} ; F ; \Gamma\right)$ and argue as above to obtain the conclusion of Theorem 5.1 .

It remains to consider the case when

$$
\frac{y_{1}(n)}{y_{2}(n)} \rightarrow \lambda \quad \text { and } \quad y_{1}(n)-\lambda y_{2}(n) \rightarrow \mu \text {. }
$$

Let $X=\lambda N_{1}+N_{2}$ and $\omega=z_{1}-\lambda z_{2}$. Then

$$
\Phi(\mathbf{z})=\exp \left(z_{2} X\right) \exp \left(\omega N_{1}\right) \gamma\left(s_{1}, s_{2}\right) F
$$

and $\omega \rightarrow \omega_{0} \in \mathcal{U}$. Clearly, $\left(X, \exp \left(\omega_{0} N_{1}\right) F\right)$ is a nilpotent orbit and, since $\left[N_{1}, \Gamma\left(0, s_{2}\right)\right]=0$, one can verify that $(3.6)$ holds and therefore

$$
\left(X ; \exp \left(\omega_{0} N_{1}\right) F, \Gamma(0, s)\right)
$$


is the asymptotic data for a period map $\hat{\Phi}$. Since $u(n) \in \Phi^{p}(\mathbf{z}(n))$ we have that

$$
u(n) \sim_{y_{2}(n)} \hat{\Phi}^{p}\left(z_{2}(n)\right) .
$$

We can then apply Proposition 5.2 to obtain that $u(n)$ takes values in a finite set

$$
\left\{v_{1}, \ldots, v_{m}\right\} \subset \operatorname{ker}(X) \cap\left(\exp \left(\omega_{0} N_{1}\right) F^{p}\right)
$$

Since $W(X)=W\left(N_{1}+N_{2}\right)$ and $\left(\exp \left(\omega_{0} N_{1}\right) F^{p}\right)$ we have shown that Theorem 5.1 holds in the last remaining case.

REMARK 6.1. If as in the last case considered above, $y_{1}(n)-\lambda y_{2}(n)$ remains bounded (the sequence $\left(y_{1}(n), y_{2}(n)\right) \in \mathbb{R}^{2}$ osculates to the line $\left.\mathbb{R}(\lambda, 1)\right)$, the elements $u(n)$ are only invariant under the action of $\lambda N_{1}+N_{2}$. This phenomenon generalizes to arbitrary $r$ and is consistent with the monodromy invariance described by Theorem 3.6. Note also that in the last case, $v_{j}$ does not necessarily lie in $F^{p}$ but it does lie in $\exp \left(\omega_{0} N_{1}\right) F^{p}$ which is also a limiting Hodge filtration.

\section{References}

1. Y. André, Pour une théorie inconditionnelle des motifs, Publications Mathématiques de l'IHES, 83, 5-49 (1996).

2. G. Ancona, Decomposition du motif d'un schema abelien universel, Thesis, math.univparis13.fr (2012).

3. M. Asakura and S. Saito, Estimate of dimension of Noether-Lefschetz locus for BeilinsonHodge cycles on open complete intersections, arXiv math/0304088, 2003

4. P. Brosnan, H. Fang, Z. Nie and G. Pearlstein, Singularities of admissible normal functions, Invent. Math. 177 599-629 (2009).

5. P. Brosnan and G. Pearlstein, The zero locus of an admissible normal function, Annals of Math. 170-2, 883-897 (2009).

6. P. Brosnan and G. Pearlstein, Zero loci of admissible normal functions with torsion singularities, Duke Math. J. 150-1, 77-100 (2009).

7. P. Brosnan, G. Pearlstein and C. Schnell, The locus of Hodge classes in an admissible variation of mixed Hodge structure, C. R., Math., Acad. Sci. Paris 348, No. 11-12, 657-660 (2010)

8. E. Cattani, P. Deligne and A. Kaplan, On the locus of Hodge classes, Jour. A.M.S., 8-2, 483-506 (1995).

9. E. Cattani and A. Kaplan, Polarized mixed Hodge structures and the local monodromy of a variation of Hodge structure, Invent. Math. 67, 101-115 (1982).

10. E. Cattani and A. Kaplan, Degenerating variations of Hodge structure, Astérisque 179-180, 67-96 (1989).

11. E. Cattani, A. Kaplan and W. Schmid, Degeneration of Hodge structures, Ann. of Math. 123, 457-535 (1986).

12. E. Cattani, A. Kaplan and W. Schmid, Variations of polarized Hodge structure: asymptotics and monodromy, Lecture Notes in Mathematics 1246, Springer-Verlag, 16-31 (1987).

13. F. Charles and C. Schnell, Notes on absolute Hodge classes. arXiv:1101.3647.

14. P. Deligne, Equations différentielles à points singuliers réguliers Lecture Notes in Mathematics 163, Springer-Verlag (1970).

15. P. Deligne, Letter to P. Griffiths.

16. P. Deligne, Travaux de Shimura, Lecture Notes in Mathematics 244, Springer (1971).

17. P. Deligne, Letter to E. Cattani and A. Kaplan (1993).

18. P. Deligne, Structures de Hodge mixtes réelles, Proc. Sympos. Pure Math., 55, Part 1, 509514, Amer. Math. Soc., Providence, RI, 1994.

19. F. Denef and G. Moore, Split States, Entropy Enigmas, Holes and Halos, J. of High Energy Physics 2011:129 (2011)

20. B. Edixhoven, On the Andr-Oort Conjecture for Hilbert Modular Surfaces, Progress in Math. 195 (2001).

21. M. Green and P. Griffiths, Algebraic cycles and singularities of normal functions II - Inspired by SS Chern, Nankai Tracts Math, 2006. 
22. P. Griffiths, Periods of integrals on algebraic manifolds, I, II, Amer. J. Math. 90, 568-626; 805-865 (1968).

23. P. Griffiths, Periods of integrals on algebraic manifolds: summary of main results and discussion of open problems. Bull. Amer. Math. Soc. 76, 228-296 (1970)

24. P. Griffiths and J. Harris, Infinitesimal variations of Hodge structure (II): an infinitesimal invariant of Hodge classes, Comp. Math. 50, 207-265 (1983).

25. P. Griffits and W. Schmid, Recent developments in Hodge theory: a discussion of techniques and results, Discrete Subgroups of Lie Groups and Applications to Moduli. Oxford University Press, 31-127 (1973)

26. A. Grothendieck, Hodge's general conjecture is false for trivial reasons, Topology 8, 299-303 (1969).

27. M. Kerr and G. Pearlstein, Boundary Components of Mumford-Tate Domains, arXiv:1210.5301 (2012)

28. B. Klingler and A. Yafaev, The André-Oort conjecture, arXiv:1209.0936 (2012).

29. R. Kloosterman, Higher Noether-Lefschetz loci of elliptic surfaces, J. Differential Geom, 76, 293-316 (2007)

30. B. Moonen, Notes on Mumford-Tate groups, unpublished notes, available at http://staff.science.uva.nl/ bmoonen/NotesMT.pdf.

31. B. Moonen and F. Oort, The Torelli locus and special subvarieties, arXiv:1112.0933.

32. A. Otwinowska, Composantes de petite codimension du lieu de Noether-Lefschetz: Un argument asymptotique en faveur de la conjecture de Hodge pour les hypersurfaces, J. Algebraic Geom. 12, 307-320 (2003)

33. C. Peters and J. Steenbrink, Monodromy of Variations of Hodge Structure, Acta Appl. Math. 75 (1-3), 183-194

34. W. Schmid, Variations of Hodge structure: the singularities of the period mapping, Invent. Math. 22, 211-319 (1973).

35. C. Simpson, The Hodge filtration on nonabelian cohomology, Proc. Symposia in Pure Math, 62-2 (1997)

36. C. Soulé and C. Voisin, Torsion cohomology classes and algebraic cycles on complex projective manifolds, Adv. Math. 198-1, 107-127 (2005)

37. C. Voisin, Hodge Loci, Handbook of Moduli, Institute de Mathematiques de Jussieu, http://www.math.jussieu.fr/ voisin/Articlesweb/hodgeloci.pdf

38. C. Voisin, Hodge loci and absolute Hodge classes, Compositio Mathematica, 143-4, 945-958, (2007).

39. A. Weil, Abelian varieties and the Hodge ring, Collected Papers III, Springer-Verlag, 421-429 (1979).

Department of Mathematics and Statistics, University of Massachusetts at Amherst, AmHerst, MA 01002, USA

E-mail address: cattani@math.umass.edu

Centro de Investigaciones y Estudios Matematicos, Universidad Nacional de Cordoba, Cordoba 5000, Argentina

E-mail address: kaplan@math.umass.edu 